University of Nebraska - Lincoln

DigitalCommons@University of Nebraska - Lincoln

6-12-2003

\title{
Interfacial edge crack between two bonded dissimilar orthotropic strips under antiplane point loading
}

Xiangfa Wu

Department of Engineering Mechanics, University of Nebraska-Lincoln, xfwu@unlserve.unl.edu

Yuris A. Dzenis

Department of Engineering Mechanics, University of Nebraska-Lincoln, ydzenis@unl.edu

Wen-Sheng Zou

Beijing Institute of Technology

Follow this and additional works at: https://digitalcommons.unl.edu/engineeringmechanicsfacpub

Part of the Mechanical Engineering Commons

Wu, Xiangfa; Dzenis, Yuris A.; and Zou, Wen-Sheng, "Interfacial edge crack between two bonded dissimilar orthotropic strips under antiplane point loading" (2003). Faculty Publications from the Department of Engineering Mechanics. 28.

https://digitalcommons.unl.edu/engineeringmechanicsfacpub/28

This Article is brought to you for free and open access by the Mechanical \& Materials Engineering, Department of at DigitalCommons@University of Nebraska - Lincoln. It has been accepted for inclusion in Faculty Publications from the Department of Engineering Mechanics by an authorized administrator of DigitalCommons@University of Nebraska - Lincoln. 
Published in ZAMM: Journal of Applied Mathematics and Mechanics / Zeitschrift für Angewandte Mathematik und Mechanik 83:6 (2003), pp. 419 - 422; doi 10.1002/zamm.200310063 Copyright @ 2003 WILEY-VCH Verlag GmbH \& Co. KGaA, Weinheim. Used by permission. http://www3.interscience.wiley.com/journal/5007542/home

Submitted December 3, 2001; revised June 24, 2002; accepted July 23, 2002; published online May 26, 2003

SHORT COMMUNICATION

\title{
Interfacial edge crack between two bonded dissimilar orthotropic strips under antiplane point loading
}

\author{
Xiang-Fa Wu, ${ }^{1}$ Yuris A. Dzenis, ${ }^{1}$ and Wen-Sheng Zou ${ }^{2}$ \\ ${ }^{1}$ Department of Engineering Mechanics, Center for Materials Research \& Analysis, \\ University of Nebraska-Lincoln, Lincoln, NE 68588-0526, USA \\ ${ }^{2}$ School of Vehicle Engineering, Beijing Institute of Technology, Beijing 100081, China \\ Corresponding author - Xiang-Fa Wu, e-mail xfwu@unlserve.unl.edu
}

\begin{abstract}
A closed-form solution is obtained for the interfacial edge crack between two bonded dissimilar orthotropic strips loaded by antiplane point loading in form of screw dislocation or line force. Conformal mapping and existing dislocation solutions are utilized for constructing the fundamental solution of the problem. The stress intensity factor (SIF) and the energy release rate (ERR) are obtained explicitly.
\end{abstract}

\section{Introduction}

Dislocation solutions play an important role in fracture mechanics as they can serve as kernel functions for crack problems. A number of investigators contributed significantly to this subject [1-4]. Finite or semi-infinite cracks in an infinite medium were considered in these papers. In practice, edge cracks are likely to occur in many materials and structures, such as composite laminates or bonded joints. The main failure mode in these materials and structures is edge delamination between the angle-layered laminas or dissimilar components [5]. Recently, interfacial edge crack problems attracted considerable attention. Shius, $\mathrm{Hu}$, and Lee [6] studied interaction between screw dislocations and an interfacial edge crack between two bonded dissimilar isotropic quarter-planes. Choi, Chong, and Chai [7] considered an interfacial edge crack between two bonded dissimilar orthotropic quarter-planes under antiplane shear. Lee and Earmme [8] examined an interfacial edge crack in an anisotropic bimaterial under antiplane singularity.

Due to complicated boundary conditions in the interfacial edge crack problems, a number of investigators used conformal mapping technique to map an edge-cracked body onto the whole plane or half-plane. The problem can then often be reduced to an existing solution for a crack in the whole plane or half plane. Suo [4] provided a general complex displacement potential for two bonded dissimilar anisotropic half-planes with a semi-infinite crack interacting with an antiplane singularity (a screw dislocation or a line force). With the displacement potential [4], an interfacial edge crack problem with complicated geometry can be solved in closed-form by finding a proper mapping function.

Most interfacial edge crack problems discussed in the literature considered an interfacial edge crack forming at the free boundary of two bonded dissimilar quarter-planes. The objective of this work is to determine the stress intensity factor $\left(\mathrm{SIF}, K_{\mathrm{III}}\right)$ and the energy release rate $\left(E R R, G_{\mathrm{III}}\right)$ for an interfacial edge crack between two bonded dissimilar orthotropic strips subjected to antiplane point loading. A closed-form solution is obtained by using conformal mapping technique and existing dislocation solutions.

\section{Formulation and solution procedure}

First, let us determine the complex displacement potential for two bonded dissimilar orthotropic strips with an interfacial edge crack subjected to antiplane singular loading in form of a screw dislocation or a line force. It is assumed that the $\xi, \eta$-plane is a mirror plane for the orthotropic materials discussed. In this case, the inplane and antiplane deformations decouple and can be treated independently. The displacement and stress components in an orthotropic elastic body subjected to an antiplane loading can be expressed in terms of an analytic function $\varphi(\zeta)$ as follows [4]:

$$
u_{3}(\xi, \eta)=-2 \operatorname{Im}[B \varphi(\zeta)], \quad \sigma_{23}(\xi, \eta)=-2 \operatorname{Re}\left[\varphi^{\prime}(\zeta)\right], \quad \sigma_{13}(\xi, \eta)=2 \operatorname{Re}\left[\mu \varphi^{\prime}(\zeta)\right]
$$


Here prime (') denotes the derivative with respect to $\zeta=\xi+\mu \eta$, and $B$ and $\mu$ are material constants defined as

$$
B=\sqrt{S_{44} S_{55}-S_{45}^{2}}, \quad \mu=\frac{S_{45}+i \sqrt{S_{44} S_{55}-S_{45}^{2}}}{S_{55}},
$$

where $S_{i j}$ are material compliances. For an orthotropic material, $S_{45}=0$ and $\mu$ is a positive imaginary variable.

Consider a semi-infinite interfacial crack between two bonded dissimilar orthotropic half-planes subjected to antiplane point loading in form of screw dislocation or line force, as shown in Figure 1(b). Without loss of generality, the singularity is located in the lower half-plane at $\zeta_{0}=\xi_{0}+\mu_{2} \eta_{0}\left(\eta_{0}<0\right)$ where $\xi$ and $\eta$ are coordinates with the origin located at the crack tip. The complex displacement potential for this antiplane problem has been obtained by Suo [4] as follows:

$$
\begin{aligned}
\varphi^{\prime}(\zeta) & =\frac{2 B_{2}}{B_{1}+B_{2}} \frac{q}{\zeta-\zeta_{0}}+\varphi_{0}^{\prime} \quad\left(\eta_{0}>0\right), \\
\varphi^{\prime}(\zeta) & =\frac{q}{\zeta-\zeta_{0}}+\bar{q} \frac{B_{2}-B_{1}}{B_{1}+B_{2}} \frac{1}{\zeta-\bar{\zeta}_{0}}+\varphi_{0}^{\prime} \quad\left(\eta_{0}<0\right),
\end{aligned}
$$

where

$$
\varphi_{0}^{\prime}(\zeta)=\frac{B_{2}}{B_{1}+B_{2}}\left\{\frac{q}{\zeta-\zeta_{0}}\left[\left(\frac{\zeta_{0}}{\zeta}\right)^{1 / 2}-1\right]+\frac{\bar{q}}{\zeta-\bar{\zeta}_{0}}\left[\left(\frac{\bar{\zeta}}{\zeta}\right)^{1 / 2}-1\right]\right\} .
$$

Here subscripts 1 and 2 denote material properties, overbar $\left(^{-}\right)$denotes the complex conjugate, and quantity $q$ is defined as

$$
q=-\frac{b}{4 \pi B_{2}}+i \frac{p}{4 \pi},
$$

where $b$ is the Burgers vector of the screw dislocation and $p$ is the line force.

The SIF and ERR can be evaluated using the following relationships:

$$
K_{\mathrm{III}}=\lim _{\xi \rightarrow 0^{+}}-2 \sqrt{2 \pi \xi} \operatorname{Re}\left[\varphi^{\prime}(\zeta)\right], \quad G_{\mathrm{III}}=\frac{1}{4}\left(B_{1}+B_{2}\right) K_{\mathrm{III}}^{2}
$$

For the particular case studied, the SIF is given by the following expression:

$$
K_{\mathrm{III}}=2 \sqrt{2 \pi} \frac{B_{2}}{B_{1}+B_{2}}\left[\frac{q}{\left(\zeta_{0}\right)^{1 / 2}}+\frac{\bar{q}}{\left(\overline{\zeta_{0}}\right)^{1 / 2}}\right] .
$$

Let us now consider an edge crack between two bonded dissimilar orthotropic strips subjected to antiplane point loading applied at $\left(x_{0}, y_{0}\right)$ as shown in Figure $1(\mathrm{a})$. Here $a$ and $W$ denote the edge crack length and the strip width, respectively, and $z=x+\mu y$ are material coordinates. Conformal mapping

$$
\varsigma=\left[\frac{g(z+a)}{g(a)}\right]^{2}-1
$$

with

$$
g(z)=\operatorname{tg}\left(\frac{\pi z}{2 W}\right)
$$

will map the edge-cracked bimaterial strips onto two bonded half-planes with a semi-infinite interfacial crack along the negative $\xi$-axis as shown in Figure 1(b). It should be noted that mapping (9) only holds when $\mu$ is a positive imaginary variable. The coordinates in the upper and lower strips are then defined as $z=x+\mu_{1} y$ and $z=x+\mu_{2} y$, respectively. Substitution of (9) into (3)-(5) yields the complex potential for the strips. Its main contributor (5) can be expressed as

$$
\begin{aligned}
\varphi_{0}^{\prime}(z)= & \left.\left.\frac{B_{2}}{B_{1}+B_{2}} \frac{2 g(z+a)\left[1+g^{2}(z+a)\right] \frac{\pi}{2 W}}{g^{2}(a)}\right\}\left\{\frac{\left[\frac{g\left(z_{0}+a\right)}{g(a)}\right]^{2}-1}{\left[\frac{g(z+a)}{g(a)}\right]^{2}-1}\right\}^{1 / 2}-1\right\} \\
& \left.\times\left\{\frac{q\left(\frac{g(z+a)}{g(a)}\right]^{2}-\left[\frac{g\left(z_{0}+a\right)}{g(a)}\right]^{2}}{\left[\frac{g(a)}{q}\right]^{2}}\left\{\frac{\left[\frac{g\left(z_{0}+a\right)}{g(a)}\right]^{2}-1}{\left[\frac{g(z+a)}{g(a)}\right]^{2}-1}\right\}^{1 / 2}-1\right\}\right\},
\end{aligned}
$$




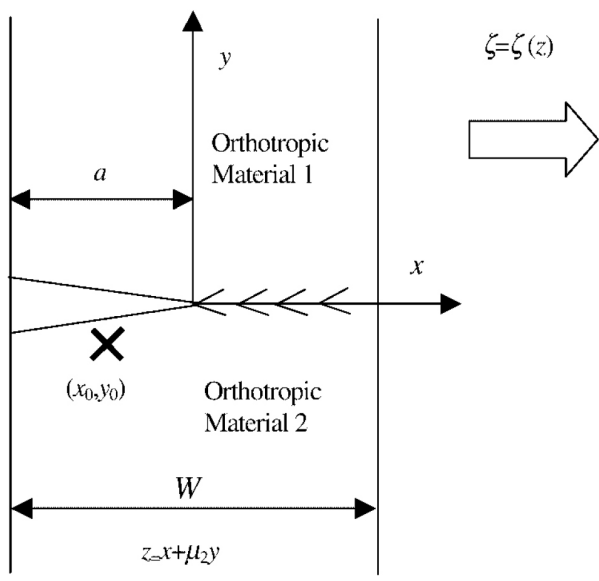

(a)

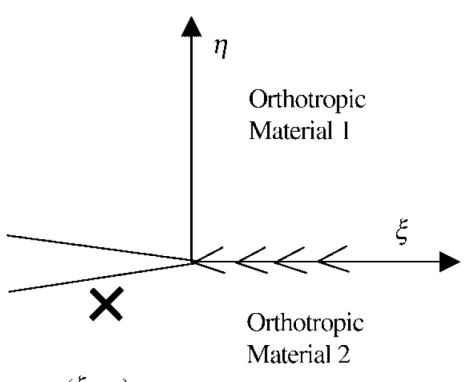

$\left(\xi_{0}, \eta_{0}\right)$

(b)

Figure 1. A cracked bimaterial under antiplane singularity: (a) an edge-cracked bimaterial strip (z-plane); (b) a semi-infinite interfacial crack between two bonded dissimilar orthotropic half-planes ( $\zeta$-plane).

where $q$ is the singularity quantity defined in (6).

Substitution of (3)-(6) and (11) into (7) yields the SIF and ERR for the problem. This solution can be used as a Green's functions to analyze edge-cracked bimaterial strips subjected to arbitrary antiplane loadings applied in the interior of the strips or at crack or strip surfaces.

\section{Example: singularity at the crack surface}

Let us consider a special case with the line force applied at the crack surface of the material 2.

Setting $q=i p / 4 \pi$ in (6) and $z_{0}=-l$ in (11) and substituting (3)-(6) and (11) into (7), we obtain the SIF of the interfacial edge crack between two bonded dissimilar orthotropic strips subjected to the line-force, $p$, located at the lower crack surface a distance $l$ behind the crack tip (see Figure 2(a)) as follows:

$$
K_{\mathrm{III}}=\lim _{x \rightarrow 0^{+}}-2 \sqrt{2 \pi x} \operatorname{Re}\left[\varphi_{2}^{\prime}(x)\right]=\frac{2 p}{\sqrt{\pi a}} \frac{B_{2}}{B_{1}+B_{2}} \sec \left(\frac{\pi a}{2 W}\right) \sqrt{\frac{\frac{\pi a}{2 W} \operatorname{tg} \frac{\pi a}{2 W}}{\operatorname{tg}^{2} \frac{\pi a}{2 W}-\operatorname{tg}^{2} \frac{\pi(a-l)}{2 W}}} .
$$

By setting $W \rightarrow \infty$ in (12), we obtain the SIF of the interfacial edge crack between two bonded dissimilar orthotropic quarter-planes (Figure 2(b)) as

$$
K_{\text {III }}=\frac{2 p}{\sqrt{\pi a}} \frac{B_{2}}{B_{1}+B_{2}} \frac{a}{\sqrt{a^{2}-(a-l)^{2}}} .
$$

The latter expression correlates with the result obtained by Lee and Earmme in [8]. It should be mentioned that, in the general anisotropic case, the mapping function $\zeta=z^{2}$ used in [8] will not map anisotropic quarter-planes onto halfplanes with a semi-infinite crack along the negative $\xi$-axis. The solution [8], therefore, holds only for the case of orthotropic materials.

Alternatively, the result (13) can be obtained by setting $W \rightarrow \infty$ in (9) and using the following mapping function: $S$ $=(z / a+1)^{2}-1$.

By setting $a \rightarrow \infty$ and keeping $c=(W-a)$ constant in (12), we obtain the SIF for a semi-infinite interfacial crack heading towards a free surface, with the lower crack surface loaded by a line force, $p$ (see Figure 2(c)) as

$$
K_{\mathrm{III}}=\frac{2 p}{\sqrt{\pi l}} \frac{B_{2}}{B_{1}+B_{2}} \frac{l+c}{\sqrt{c(l+2 c)}} .
$$

By further setting $c \rightarrow \infty$ in (14), we obtain the SIF for a semi-infinite interfacial crack between two bonded dissimilar orthotropic half-planes as

$$
K_{\text {III }}=\sqrt{\frac{2}{\pi l}} \frac{B_{2}}{B_{1}+B_{2}} p .
$$

The latter relation coincides with the result given by Suo [4]. 


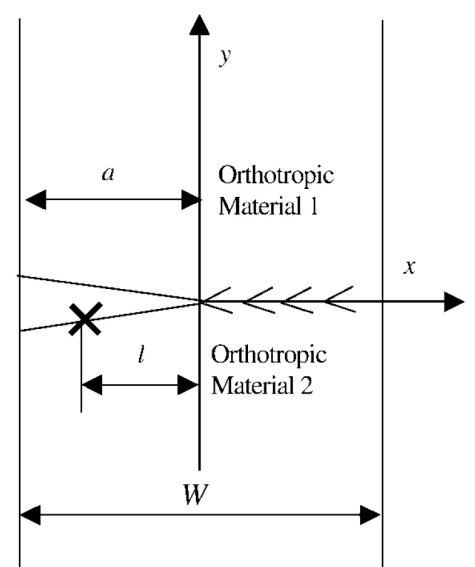

(a)

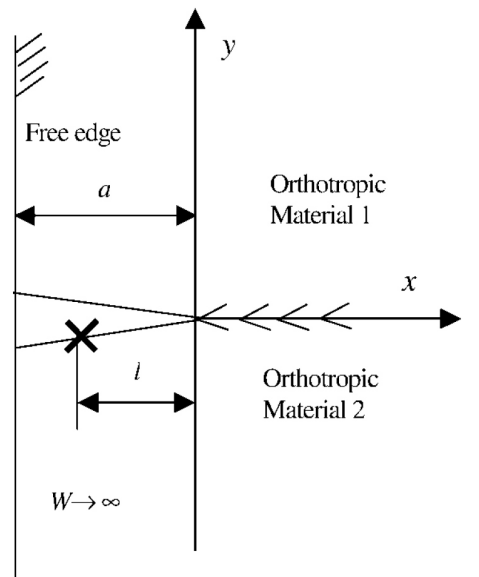

(b)

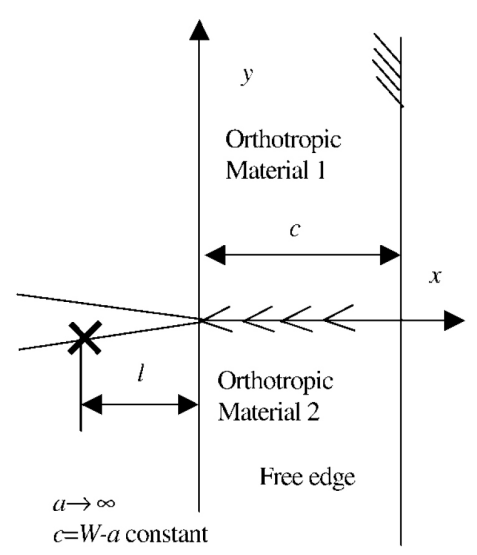

(c)

Figure 2. A cracked bimaterial with an antiplane point loading applied on the lower crack surface: (a) an edge-cracked bimaterial strip; (b) an interfacial crack between two bonded dissimilar anisotropic quarter-planes; (c) a semi-infinite interfacial crack heading towards a free surface.

The ERR for all crack configurations discussed can be easily obtained by inserting (12)-(15) into (7).

With $B_{1}=B_{2}$, the above results cover solutions for the edge-cracked homogeneous orthotropic (or isotropic) strips or half-planes.

\section{Conclusions}

With proper selection of conformal mapping function, we obtained the closed-form solutions for the complex displacement potential, SIF $\left(K_{\mathrm{III}}\right)$, and ERR $\left(G_{\mathrm{III}}\right)$ for two bonded dissimilar orthotropic strips with an interfacial edge crack subjected to antiplane point loading. The formulas presented can be easily modified for the edge-cracked homogeneous strips or interfacial edge cracks between two bonded strips, quarter-planes, or half-planes made of dissimilar isotropic materials. Furthermore, the obtained closed-form results can be used as Green's functions to analyze SIF and ERR for edge-cracked strips loaded with arbitrary antiplane loadings applied either in the interior of the strips or on crack or strip surfaces. An alternative analysis of the latter problems by dual integral equations would generally be more difficult.

\section{Acknowledgments}

The support of this work by the U.S. Army Research Office is gratefully acknowledged. The authors would like to thank the anonymous reviewers for ZAMM for helpful comments.

\section{References}

[1] J. R. Rice and R. Thomson, Ductile versus brittle behavior of crystals, Philos. Mag. 29, 73-97 (1974).

[2] S. M. Ohr, An electron microscope study of crack tip deformation and its impact on the dislocation theory of fracture, Mater. Sci. Eng. 72, 1-35 (1985).

[3] R. Thomson, Physics of fracture, Solid State Phys. 39, 1-129 (1986).

[4] Z. Suo, Singularities, interfaces and cracks in dissimilar anisotropic media, Proc. R. Soc. Lond. A 427, 331-358 (1990).

[5] M. R. Jones, Mechanics of Composite Materials, 2nd ed. (Taylor \& Francis, Philadelphia, USA, 1999).

[6] S. T. Shiue, C. T. Hu, and S. Lee, Elastic interaction between screw dislocations and a welded surface crack in composite materials, Eng. Fract. Mech. 33(5), 697-706 (1989).

[7] S. R. Choi, C. H. Chong, and Y. S. Chai, Interfacial edge crack in two bonded dissimilar orthotropic quarter planes under antiplane shear, Int. J. Fract. 67, 143-150 (1994).

[8] K. W. Lee and Y. Y. Earmme, An interfacial edge crack in anisotropic bimaterial under antiplane singularity, Int. J. Fract. 104, 15$22(2000)$. 